Pacific Journal of Mathematics

REMOVABLE SINGULARITIES FOR SUBHARMONIC 


\title{
REMOVABLE SINGULARITIES FOR SUBHARMONIC FUNCTIONS
}

\author{
STEPHEN J. Gardiner
}

Let $\Omega$ be an open set in $\mathbb{R}^{n}(n \geq 3)$ and $S$ be a $C^{2}(n-1)$ dimensional manifold in $\Omega$. Let $\alpha \in(0, n-2)$ and $E$ be a compact subset of $S$ of zero $\alpha$-dimensional Hausdorff measure. We show that, if $s$ is subharmonic in $\Omega \backslash E$ and satisfies $s(X) \leq c[\operatorname{dist}(X, S)]^{\alpha+2-n}$ for $X \in \Omega \backslash S$, then $s$ has a subharmonic extension to the whole of $\Omega$. The sharpness of this and other similar results is also established.

1. Introduction and results. Let $\Omega$ denote an open set in Euclidean space $\mathbb{R}^{n}(n \geq 3)$, and let $E$ be a compact subset of $\Omega$. This paper is concerned with results of the following type: if $s$ is a subharmonic function in $\Omega \backslash E$, where $E$ is "small" and $s$ is "not too badly behaved" (near $E$ ), then $s$ has a subharmonic extension to the whole of $\Omega$. We say in this case that $E$ is a removable singularity of $s$. There is an obvious analogue of this notion for harmonic functions.

It is a consequence of a classical result [7, Theorem 5.18] that, if $E$ is polar and $s$ is a subharmonic function on $\Omega \backslash E$ which is bounded above near $E$, then $E$ is a removable singularity of $s$. The idea behind our results is that, by imposing constraints on the geometry and size of the set $E$, the boundedness requirement can be considerably relaxed. The size of $E$ is measured in terms of its $\alpha$-dimensional Hausdorff measure $m_{\alpha}(E)$. A discussion of Hausdorff measures in relation to subharmonic functions can be found in Hayman and Kennedy [7, $\S 5.4]$.

Let $O_{n}$ denote the origin of $\mathbb{R}^{n}$, let $|X|$ denote the Euclidean norm of a point $X \in \mathbb{R}^{n}$, and $B(X, r)$ be the open ball of centre $X$ and radius $r$. Also, let $\Phi: \Omega \rightarrow \mathbb{R}$ be a $C^{2}$ function with nonvanishing gradient throughout $\Omega$. We put $S=\{Y \in \Omega$ : $\Phi(Y)=0\}$.

Theorem 1. Let $\alpha \in(0, n-2)$ and $E$ be a compact subset of $S$ such that $m_{\alpha}(E)=0$. If $s$ is subharmonic in $\Omega \backslash E$ and satisfies

$$
s(X) \leq c[\operatorname{dist}(X, S)]^{\alpha+2-n} \quad(X \in \Omega \backslash S)
$$

for some positive constant $c$, then $E$ is a removable singularity of $s$. 
Corollary. Let $\alpha$ and $E$ be as above. If $h$ is harmonic in $\Omega \backslash E$ and satisfies

$$
|h(X)| \leq c[\operatorname{dist}(X, S)]^{\alpha+2-n} \quad(X \in \Omega \backslash S),
$$

then $E$ is a removable singularity of $h$.

Regarding the bound on $\alpha$, Theorem 1 is true, but not interesting, when $\alpha=n-2$ : for then we are requiring $s$ to be bounded above near the set $E$, which is polar by [7, Theorem 5.14]. For higher values of $\alpha$, the set $E$ need not be polar [7, Theorem 5.13].

Theorem 1 is related to work by Dahlberg [4] on subharmonic functions in Lipschitz domains. In the case of a domain with a $C^{2}$ boundary, his theorem simplifies to the following boundary analogue of Theorem 1.

TheOREM A. Let $\Omega$ be a bounded domain in $\mathbb{R}^{n}$ with $C^{2}$ boundary, let $\alpha \in(0, n-1)$, and let $E$ be a closed subset of $\partial \Omega$ such that $m_{\alpha}(E)=0$. If $s$ is subharmonic in $\Omega$, satisfies $\lim \sup s(X) \leq 0$ as $X \rightarrow Y \in \partial \Omega \backslash E$, and

$$
s(X) \leq c[\operatorname{dist}(X, \partial \Omega)]^{\alpha+1-n} \quad(X \in \Omega),
$$

then $s \leq 0$ in $\Omega$.

The sharpness of Theorem 1 and its corollary is shown by the following result, which does not require $E$ to be a subset of $S$. (Clearly (2) is stronger than (1) when $E \subset S$.)

THEOREM 2. Let $\alpha \in(0, n-2)$ and $E$ be a compact set such that $m_{\alpha}(E)>0$. Then there is a positive harmonic function $h$ on $\mathbb{R}^{n} \backslash E$ such that

$$
h(X) \leq[\operatorname{dist}(X, E)]^{\alpha+2-n} \quad\left(X \in \mathbb{R}^{n} \backslash E\right),
$$

but for which $E$ is not a removable singularity.

It is natural to ask whether Theorem 1 remains true if we drop the requirement $E \subset S$ and replace (1) by (2). The following example shows that this is far from the case.

EXAMPLE. For each $k \in \mathbb{N}$ let $S_{k}=\partial B\left(O_{n},[\log (k+1)]^{-1}\right)$ and let $E_{k}$ be a finite subset of $S_{k}$ such that $B(X, 1 / k) \cap E_{k}$ is non-empty for any $X \in S_{k}$. Then the compact set $E=\left[\bigcup_{k} E_{k}\right] \cup\left\{O_{n}\right\}$ has the property that $m_{\alpha}(E)=0$ for every $\alpha>0$. On the other hand, the 
function $s(X)=|X|^{2-n}$ is harmonic on $\mathbb{R}^{n} \backslash E$ and is easily seen to satisfy

$$
s(X) \leq C(n, \alpha)[\operatorname{dist}(X, E)]^{\alpha+2-n} \quad\left(X \in \mathbb{R}^{n} \backslash E\right)
$$

for any $\alpha \in[0, n-2)$.

Among previous work on removable singularities of subharmonic functions we mention papers by Shapiro [14], Kuran [11], Kaufman and $\mathrm{Wu}$ [9], and Armitage [1]. Our results are new in that, by introducing the restriction $E \subset S$, we are able to permit very bad behaviour of $s$ near $E$. Thus, for example, the Lebesgue integrability requirement in [14] is not applicable in Theorem 1 if $\alpha \leq n-3$. We mention also Cima and Graham [3], who showed that an analytic subvariety $E$ in the unit ball of $\mathbb{C}^{n}$ is a removable singularity for holomorphic functions which satisfy appropriate growth conditions near $E$.

A slight modification of the proof of Theorem 1 yields the following.

THEOREM 3. Let $\alpha \in(0, n-2)$ and $E$ be a compact subset of $S$ such that $m_{\alpha}(E)<+\infty$. If $s$ is subharmonic in $\Omega \backslash E$ and satisfies

$$
s(X) \leq u(\operatorname{dist}(X, S)) \quad(X \in \Omega \backslash S)
$$

where $t^{n-\alpha-2} u(t) \rightarrow 0(t \rightarrow 0+)$, then $E$ is a removable singularity of $s$.

Theorem 3 can be regarded as a generalization of the following simple, well-known fact, which corresponds to the case $\alpha=0:$ if $s$ is subharmonic in $\Omega \backslash\{Y\}$ and limsup $|X-Y|^{n-2} s(X) \leq 0$ as $X \rightarrow Y$, then $\{Y\}$ is a removable singularity of $s$. The following shows that Theorem 3 is sharp.

THEOREM 4. Let $\alpha \in(0, n-2)$ and $E$ be a compact set which is not $\sigma$-finite with respect to $m_{\alpha}$. Then there is a positive harmonic function $h$ on $\mathbb{R}^{n} \backslash E$ such that

$$
\sup \{h(X): \operatorname{dist}(X, E)=\rho\}=o\left(\rho^{\alpha+2-n}\right) \quad(\rho \rightarrow 0+),
$$

but for which $E$ is not a removable singularity.

Using ideas from [12], we can apply Theorem A to give removability results based on the behaviour of the means, $\mathscr{A}\left(s^{+} ; X, r\right)$ and $\mathscr{M}\left(s^{+} ; X, r\right)$, of $s^{+}$over the ball $B(X, r)$ and the sphere $\partial B(X, r)$ respectively. (Given $X \in \Omega$, the function $s^{+}$will be defined at least almost everywhere on $\partial B(X, r)$ for all small $r>0$.) The following 
theorem, which is close to a result of Shapiro [14], complements removable singularity results of Armitage [1] based on the behaviour of spherical means.

THEOREM 5. Let $\alpha \in(0, n-2)$ and $E$ be a compact subset of $\Omega$ such that $m_{\alpha}(E)=0$. If $s$ is subharmonic in $\Omega \backslash E$ and satisfies

$$
\mathscr{A}\left(s^{+} ; X, r\right) \leq c r^{\alpha+2-n} \quad(\overline{B(X, r)} \subset \Omega),
$$

then $E$ is a removable singularity of $s$.

THEOREM 6. Let $\alpha \in(0, n-2)$ and $E$ be a compact subset of $\mathbb{R}^{n}$ such that $m_{\alpha}(E)>0$. Then there is a positive harmonic function $h$ on $\mathbb{R}^{n} \backslash E$ such that

$$
\mathscr{M}(h ; X, r) \leq r^{\alpha+2-n} \quad\left(X \in \mathbb{R}^{n}\right),
$$

but for which $E$ is not a removable singularity.

It is a pleasure to thank Professor Anthony O'Farrell for a number of stimulating discussions.

\section{Proofs of Theorems 1 and 3.}

2.1. Let $\varphi \in(0, \pi / 2)$, let $X=\left(x, X^{\prime}\right) \in \mathbb{R} \times \mathbb{R}^{n-1}$, and define

$$
D(Q, r)=\left\{X \in \overline{B(Q, 2 r)}:\left|X^{\prime}-Q^{\prime}\right| \tan \varphi \leq|x-q|\right\}
$$

for $Q \in \mathbb{R}^{n}$ and $r>0$.

Lemma A. On the set $R=\left\{X:|x|<\left|X^{\prime}\right| \tan \varphi\right\}$ there is a positive harmonic function of the form

$$
|X|^{k(\varphi)} F_{\varphi}\left(\tan ^{-1}\left(|x| /\left|X^{\prime}\right|\right)\right),
$$

where $k(\varphi)>0, F_{\varphi}(\varphi)=0$ and $F_{\varphi}^{\prime}$ is continuous on $(0, \pi / 2)$. Further, $k(\varphi) \rightarrow \infty$ as $\varphi \rightarrow 0+$.

It is well known that there is a positive harmonic function $h$ on $R$ of the form $|X|^{k(\varphi)} F(X /|X|)$, where $k(\varphi)>0$ and $F=0$ on $\partial R \cap \partial B\left(O_{n}, 1\right)$. Further, $F$ is unique up to a multiplicative constant. This uniqueness and the symmetry properties of $R$ imply that $h$ has the form (4), where $F_{\varphi}(\varphi)=0$. Consideration of the second order ordinary differential equation satisfied by $F_{\varphi}$ shows that $F_{\varphi} \in C^{\infty}(0, \pi / 2)$. For the final assertion, see Friedland and Hayman [6]. 
We now fix $\varphi$ small enough to ensure that $k(\varphi) \geq n-1$. The set $R \cap B\left(O_{n}, 1\right)$ is a NTA domain in the sense of Jerison and Kenig [8], so the boundary Harnack principle $[8,(5.1)]$ can be applied to show that there is a positive constant $C(n, \varphi)$ such that the Green kernel $G$ for the set $\mathbb{R}^{n} \backslash D\left(O_{n}, 1\right)$ satisfies

$$
G(X, Y) \leq C(n, \varphi)|X|^{k(\varphi)} F_{\varphi}\left(\tan ^{-1}|x| /\left|X^{\prime}\right|\right)
$$

for

$$
X \in B\left(O_{n}, 1\right) \backslash D\left(O_{n}, 1\right) \text { and } Y \in \mathbb{R}^{n} \backslash B\left(O_{n}, 3\right) .
$$

2.2. Let $L=\left\{X^{\prime} \in \mathbb{R}^{n-1}:\left|X^{\prime}\right|<4\right\}$ and $f: L \rightarrow \mathbb{R}$ be a $C^{2}$ function such that $f\left(O_{n-1}\right)=0$ and $\left|\nabla f\left(O_{n-1}\right)\right|=0$. We write

$$
\left|\nabla_{2} f\right|=\left\{\sum_{j, k=1}^{n-1}\left[\frac{\partial^{2} f}{\partial x_{j} \partial x_{k}}\right]^{2}\right\}^{1 / 2} .
$$

Further, let $T=\left\{\left(f\left(X^{\prime}\right), X^{\prime}\right): X^{\prime} \in L\right\}$ and put

$$
A=\bigcup_{\left\{Q \in T:\left|Q^{\prime}\right| \leq 1\right\}} D(Q, 1)
$$

A simple sequence argument shows that $A$ is a closed set.

If $J \subset \mathbb{R}^{n}$ is compact, we use $H[J, F]$ to denote the PerronWiener-Brelot solution to the generalized Dirichlet problem for the unbounded component of $\mathbb{R}^{n} \backslash J$, with data $F$ on the finite boundary and 0 at infinity.

LeMmA 1. Let $\beta \in(0, n-2)$ and define $g$ on $\partial A$ by

$$
g(X)= \begin{cases}{[\operatorname{dist}(X, T)]^{-\beta}} & (X \notin T), \\ 0 & (X \in T) .\end{cases}
$$

There are positive constants $\eta, K$ such that, if $f$ is as above and satisfies $\left|\nabla_{2} f\right|<\eta$ on $L$, then $g$ is integrable with respect to harmonic measure for $\mathbb{R}^{n} \backslash A$ and

$$
H[A, g](Y) \leq K|Y|^{2-n} \quad(|Y|>5) .
$$

To prove this, let $\eta$ be sufficiently small to ensure that $\left|f\left(Q^{\prime}\right)\right| \leq \sqrt{3}$ whenever $\left|Q^{\prime}\right| \leq 1$. Thus $|X| \leq 4$ for $X \in A$. Let $G_{*}$ be the Green kernel for the set $\mathbb{R}^{n} \backslash A$, and let $G_{Q}$ be the Green kernel for the set $\mathbb{R}^{n} \backslash D(Q, 1)$, where $Q$ is some point in $T$ satisfying $\left|Q^{\prime}\right|=1$. Then $G_{*} \leq G_{Q}$ in $\mathbb{R}^{n} \backslash A$, and so from $\S 2.1$,

$$
G_{*}(X, Y) \leq C(n, \varphi)|X-Q|^{k(\varphi)} F_{\varphi}\left(\tan ^{-1}\left|x-q \| X^{\prime}-Q^{\prime}\right|\right)
$$


for

$$
X \in B(Q, 1) \backslash A \text { and } Y \in \mathbb{R}^{n} \backslash B\left(O_{n}, 5\right) .
$$

Dividing by $\operatorname{dist}(X, A)$ and taking limits along the normal common to $\partial A$ and $\partial D(Q, 1)$, we see that (for small $\eta$ ) the normal derivative $\partial G_{*} / \partial n_{X}$ at $X$ satisfies

$$
\frac{\partial G_{*}}{\partial n_{X}}(X, Y) \leq C(n, \varphi, \eta)[\operatorname{dist}(X, T)]^{k(\varphi)-1},
$$

where

$$
X \in\{Z \in \partial A: 0<\operatorname{dist}(X, T)<(\sin \varphi) / 2\} \quad \text { and } \quad Y \in \mathbb{R}^{n} \backslash B\left(O_{n}, 5\right) \text {. }
$$

Since $k(\varphi)-1>n-2>\beta$, it now follows that $g$ is integrable with respect to harmonic measure for $\mathbb{R}^{n} \backslash A$. Further, the surface area of $\{X \in \partial A: \operatorname{dist}(X, T)<(\sin \varphi) / 2\}$ is bounded above by a constant depending on $n, \varphi, \eta$, but not $f$, so we can write

$$
H[A, g](Y) \leq C(n, \varphi, \eta) \quad(|Y|=5),
$$

and hence

$$
H[A, g](Y) \leq C(n, \varphi, \eta)(5 /|Y|)^{n-2} \quad(|Y| \geq 5) .
$$

(Note: Dahlberg [5] has shown that, for bounded Lipschitz domains, harmonic measure is absolutely continuous with respect to surface area measure, and that the density function is given by the normal derivative of the Green function. In the above argument we have used this fact and the observation that the image of $\mathbb{R}^{n} \backslash A$ under inversion in $\partial B\left(O_{n}, 1\right)$ is a bounded Lipschitz domain, punctured at the origin.)

2.3. We now complete the proof of Theorem 1. From the implicit function theorem there exists $\gamma>0$ such that, if $Z \in E$, then (choosing a suitable coordinate system $\left(\tilde{x}_{1}, \ldots, \tilde{x}_{n}\right)$ centred at $\left.Z\right)$ there is a $C^{2}$ function $f_{Z}$ such that $f_{Z}\left(\widetilde{O}_{n-1}\right)=0,\left|\nabla f_{Z}\left(\widetilde{O}_{n-1}\right)\right|=0$ and

$$
\left\{X \in S:|\tilde{x}|<\gamma \text { and }\left|\tilde{X}^{\prime}\right|<\gamma\right\}=\left\{X:\left|\tilde{X}^{\prime}\right|<\gamma \text { and } \tilde{x}=f_{Z}\left(\tilde{X}^{\prime}\right)\right\} \text {. }
$$

Let $\varepsilon>0$ and $0<\delta<\gamma / 4$. Since $m_{\alpha}(E)=0$, there exists a finite collection of open balls $B_{i}$ of radii $r_{i} / 2<\delta / 2$ such that

$$
E \subset \bigcup_{i} B_{i} \text { and } \sum_{i} r_{i}^{\alpha}<\varepsilon
$$

For each $i$, choose $Z_{i} \in B_{i} \cap E$ (any $B_{i}$ for which $B_{i} \cap E$ is empty is discarded) and, using the above coordinate system centred at $Z_{i}$, put

$$
A_{i}=\bigcup_{\left\{Q \in S:\left|\widetilde{Q}^{\prime}\right| \leq r_{\imath}\right\}} \widetilde{D}\left(Q, r_{i}\right)
$$


Clearly $E \subset \bigcup_{i} A_{i}$ if $\delta$ is small. We define $g_{i}$ on $\partial A_{i}$ by

$$
g_{i}(X)= \begin{cases}{[\operatorname{dist}(X, S)]^{\alpha+2-n}} & (X \notin S), \\ 0 & (X \in S) .\end{cases}
$$

Applying a dilation of centre $Z_{i}$ and magnification factor $r_{i}$, it follows from Lemma 1 that

$$
H\left[A_{i}, g_{i}\right](Y) \leq K r_{i}^{\alpha}\left|Y-Z_{i}\right|^{2-n} \quad\left(Y \in \mathbb{R}^{n} \backslash B\left(Z_{i}, 5 r_{i}\right)\right) .
$$

(Note that, provided $\gamma>0$ is chosen small enough, the hypothesis $\left|\nabla_{2} f\right|<\eta$ is satisfied.)

Now let $V$ be a bounded open set such that $E \subset V \subset \bar{V} \subset \Omega$ and let $a$ be an upper bound for $s$ on $\partial V$. If $X \in V \backslash \bigcup_{i} B\left(Z_{i}, 5 \delta\right)$, then by (1), (5) and (6),

$$
\begin{aligned}
(s-a)^{+}(X) & \leq c \sum_{i} H\left[A_{i}, g_{i}\right](X) \\
& \leq c K \sum_{i} r_{i}^{\alpha}\left|X-Z_{i}\right|^{2-n} \\
& \leq c K(5 \delta)^{2-n} \varepsilon .
\end{aligned}
$$

Since $\varepsilon>0$ can be arbitrarily small, we have $s(X) \leq a$ for $X \in V$ satisfying $\operatorname{dist}(X, E) \geq 5 \delta$. Further, since $\delta>0$ can be arbitrarily small, we have $s \leq a$ in $V \backslash E$. Thus $s$ is bounded above near the polar set $E$, and so $E$ is a removable singularity of $s$.

2.4. The proof of Theorem 3 requires only self-evident modification to (5) and the last paragraph of $\S 2.3$.

\section{Proofs of Theorems 2 and 4.}

3.1. Theorem 2 relies on a lemma due to Frostman [7, Lemma 5.4]. This says that, if $m_{\alpha}(E)>0$, then there is a finite, positive measure $\mu$ on $E$ such that $\mu(B(X, r)) \leq r^{\alpha}$ for any ball $B(X, r)$. Clearly we can assume that $\mu(E) \leq 1$. The Newtonian potential $v$, due to $\mu$, is harmonic on $\mathbb{R}^{n} \backslash E$ but not on $\mathbb{R}^{n}$.

Now let $X \in \mathbb{R}^{n} \backslash E$ and $\rho=\operatorname{dist}(X, E)$. If $\rho<1$ and $Y \in$ $B(X, \rho)$, then integration by parts yields

$$
\begin{aligned}
v(Y) & =\int_{E}|Y-Z|^{2-n} d \mu(Z) \\
& \leq(n-2) \int_{\rho-|Y-X|}^{\infty} \mu(B(Y, t)) t^{1-n} d t \\
& \leq(n-2) \int_{\rho-|Y-X|}^{\infty} \min \left\{t^{\alpha}, 1\right\} t^{1-n} d t \\
& \leq(n-2)(n-2-\alpha)^{-1}(\rho-|Y-X|)^{\alpha+2-n} .
\end{aligned}
$$


Putting $p=[2(n-2-\alpha)]^{-1}$, we now have

$$
\begin{aligned}
\left\{\rho^{-n} \int_{B(X, \rho)}[\right. & \left.v(Y)]^{p} d Y\right\}^{1 / p} \\
& \leq C(n, \alpha)\left\{\rho^{-n} \int_{0}^{\rho} t^{n-1}[\rho-t]^{(\alpha+2-n) p} d t\right\}^{1 / p} \\
& \leq C(n, \alpha)\left\{2 \rho^{-1 / 2}\right\}^{1 / p} \\
& \leq C(n, \alpha) \rho^{\alpha+2-n} .
\end{aligned}
$$

Applying an inequality originally due to Hardy and Littlewood in the case $n=2$, and extended by Kuran to higher dimensions [10, Theorem 1], it follows that

$$
v(X) \leq C(n, \alpha) \rho^{\alpha+2-n},
$$

and so, letting $h=v / C(n, \alpha)$, we obtain (2).

3.2. To prove Theorem 4 , we note (see $[13, \mathrm{pp} .83,84]$ ) that, if $E$ is not $\sigma$-finite with respect to $m_{\alpha}$, then there is a positive, nondecreasing, continuous function $w$ on $[0,+\infty)$ such that $t^{-\alpha} w(t) \rightarrow$ $0(t \rightarrow 0+)$ and $E$ is not $\sigma$-finite with respect to $m_{w}$. (Here $m_{w}$ refers to the Hausdorff measure generated by $w$.) As in $\S 3.1$ there exists a finite, positive measure $\mu$ on $E$ such that $\mu(B(X, r)) \leq w(r)$, for any ball $B(X, r)$. We write $h$ for the Newtonian potential due to $\mu$ and assume that $\mu(E) \leq 1$.

Now let $\varepsilon>0$ and choose $\delta>0$ such that $t^{-\alpha} w(t) \leq \varepsilon$ for $t \in(0, \delta)$. Also, let $X \in \mathbb{R}^{n} \backslash E$ and $\rho=\operatorname{dist}(X, E)$. If $\rho<\delta$ and $Y \in B(X, \rho)$, the reasoning of $\S 3.1$ yields

$$
h(Y) \leq \delta^{2-n}+\varepsilon(n-2)(n-2-\alpha)^{-1}(\rho-|Y-X|)^{\alpha+2-n}
$$

and

$$
h(X) \leq C(n, \alpha)\left(\delta^{2-n}+\varepsilon \rho^{\alpha+2-n}\right),
$$

whence

$$
\limsup _{\rho \rightarrow 0+}\left(\rho^{n-2-\alpha} \sup \{h(X): \operatorname{dist}(X, E)=\rho\}\right) \leq \varepsilon C(n, \alpha) .
$$

Since $\varepsilon>0$ can be arbitrarily small, the result follows.

\section{Proofs of Theorems 5 and 6.}

4.1. To prove Theorem 5, let $V$ be a bounded open set such that $E \subset V \subset \bar{V} \subset \Omega$ and let $a$ be an upper bound for $s$ on $\partial V$. Let $v=(s-a)^{+}$on $V$ and let $v=0$ on $\mathbb{R}^{n} \backslash V$. Clearly $v$ is 
subharmonic on $\mathbb{R}^{n} \backslash E$. Now let $I_{v}$ denote the Poisson integral of $v$ in $\mathbb{R}^{n} \times(0,+\infty)$. Using integration by parts and then (3), it follows that

$$
\begin{aligned}
I_{v}(X, y) & =C(n) y \int_{0}^{\infty} t^{n+1}\left(y^{2}+t^{2}\right)^{-(n+3) / 2} \mathscr{A}(v ; X, t) d t \\
& \leq C(n, \alpha, c) y^{\alpha+2-n} \quad\left(X \in \mathbb{R}^{n} ; y>0\right) .
\end{aligned}
$$

Applying Harnack's inequalities [2, p. 200] twice in the ball of centre $(X, y)$ and radius $y / 2$, we have

$$
I_{(-\Delta v)}(X, y)=\partial^{2} I_{v}(X, y) / \partial y^{2} \leq C(n, \alpha, c) y^{\alpha-n} .
$$

But the distributional Laplacian $\Delta v$ is non-negative on $\mathbb{R}^{n} \backslash E$ by the subharmonicity of $v$. Applying a half-space version of Theorem $\mathrm{A}$ (obtained by inversion from the corresponding result for the ball), it follows that $I_{(-\Delta v)} \leq 0$ on $\mathbb{R}^{n} \times(0,+\infty)$. Hence $\Delta v \geq 0$ on $\mathbb{R}^{n}$ and so $v$ has a subharmonic extension to $\mathbb{R}^{n}$. Since $s$ is now bounded above on $V \backslash E$, it follows that the polar set $E$ is a removable singularity of $s$.

4.2. Let $\mu$ and $v$ be as in the proof of Theorem 2. Then (cf. [7, $(3.9 .6)])$

$$
\begin{aligned}
\mathscr{M}(v ; X, r) & =(n-2) \int_{r}^{\infty} t^{1-n} \mu(B(X, t)) d t \\
& \leq(n-2) \int_{r}^{\infty} t^{\alpha+1-n} d t=C(n) r^{\alpha+2-n},
\end{aligned}
$$

and Theorem 6 follows.

\section{REFERENCES}

[1] D. H. Armitage, Mean values and associated measures of superharmonic functions, Hiroshima Math. J., 13 (1983), 53-63.

[2] M. Brelot, Élements de la théorie classique du potentiel, Centre de documentation universitaire, Paris, 1965.

[3] J. A. Cima and I. R. Graham, On the extension of holomorphic functions with growth conditions across analytic subvarieties, Michigan Math. J., 28 (1981), 241-256.

[4] B. E. J. Dahlberg, On exceptional sets at the boundary for subharmonic functions, Ark. Mat., 15 (1977), 305-312.

[5] _ Estimates of harmonic measure, Arch. Rational Mech. Anal., 65 (1977), 275-288.

[6] S. Friedland and W. K. Hayman, Eigenvalue inequalities for the Dirichlet problem on spheres and the growth of subharmonic functions, Comment. Math. Helvetici, 51 (1976), 133-161. 
[7] W. K. Hayman and P. B. Kennedy, Subharmonic functions, Volume 1, Academic Press, London, 1976.

[8] D. S. Jerison and C. E. Kenig, Boundary behaviour of harmonic functions in non-tangentially accessible domains, Advances in Math., 46 (1982), 80-147.

[9] R. Kaufman and J.-M. Wu, Removable singularities for analytic or subharmonic functions, Ark. Mat., 18 (1980), 107-16. Correction, ibid., 21 (1983), 1.

[10] Ü. Kuran, Subharmonic behaviour of $|h|^{p}(p>0, h$ harmonic), J. London Math. Soc. (2), 8 (1974), 529-538.

[11] _ Some extension theorems for harmonic, superharmonic and holomorphic functions, J. London Math. Soc. (2), 22 (1980), 269-284.

[12] A. G. O'Farrell, The 1-reduction for removable singularities, and the negative Hölder spaces, Proc. Roy. Irish Acad., 88A (1988), 133-151.

[13] C. A. Rogers, Hausdorff Measures, Cambridge University Press, London, 1970.

[14] V. L. Shapiro, Subharmonic functions and Hausdorff measure, J. Differential Equations, 27 (1978), 28-45.

Received February 15, 1989.

UNiversity College

Belfield, Dublin 4

IRELAND 


\section{PACIFIC JOURNAL OF MATHEMATICS EDITORS}

V. S. VARADARAJAN

(Managing Editor)

University of California

Los Angeles, CA 90024-1555-05

Herbert Clemens

University of Utah

Salt Lake City, UT 84112

THOMAS ENRIGHT

University of California, San Diego

La Jolla, CA 92093

\section{R. FINN}

Stanford University

Stanford, CA 94305

HermanN FlaschKa

University of Arizona

Tucson, AZ 85721

VAUGHAN F. R. JoNES

University of California

Berkeley, CA 94720

STEVEn KeRCKHOFF

Stanford University

Stanford, CA 94305
C. C. MOORE

University of California

Berkeley, CA 94720

MARTIN SCHARLEMANN

University of California

Santa Barbara, CA 93106

Harold STARK

University of California, San Diego La Jolla, CA 92093

\section{ASSOCIATE EDITORS}

R. ARENS

E. F. BECKENBACH (1906-1982)
B. H. NeumanN

\section{SUPI}

UNIVERSITY OF ARIZONA

UNIVERSITY OF BRITISH COLUMBIA

CALIFORNIA INSTITUTE OF TECHNOLOGY

UNIVERSITY OF CALIFORNIA

MONTANA STATE UNIVERSITY

UNIVERSITY OF NEVADA, RENO

NEW MEXICO STATE UNIVERSITY

OREGON STATE UNIVERSITY
F. WolF
K. YoshidA
(1904-1989)

UNIVERSITY OF OREGON UNIVERSITY OF SOUTHERN CALIFORNIA

STANFORD UNIVERSITY

UNIVERSITY OF HAWAII

UNIVERSITY OF TOKYO

UNIVERSITY OF UTAH

WASHINGTON STATE UNIVERSITY

UNIVERSITY OF WASHINGTON 


\section{Pacific Journal of Mathematics}

Vol. 147, No. $1 \quad$ January, 1991

Mark S. Ashbaugh, Evans Malott Harrell, II and Roman Svirsky, On minimal and maximal eigenvalue gaps and their causes $\ldots \ldots \ldots \ldots \ldots 1$

Robert Coleman and Francis Oisin McGuinness, Rational formal group

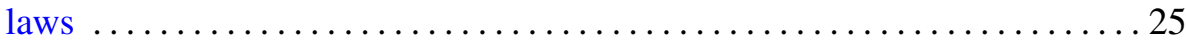

Jacek M. Cygan and Leonard Frederick Richardson, $D$-harmonic distributions and global hypoellipticity on nilmanifolds ...........29

Satya Deo and Kalathoor Varadarajan, Some examples of nontaut

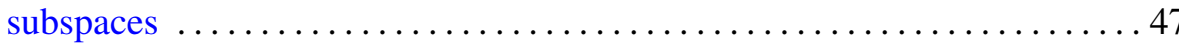

Maria Fragoulopoulou, Automatic continuity of *-morphisms between

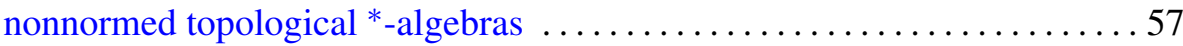

Stephen J. Gardiner, Removable singularities for subharmonic functions . . . 71 Herbert Paul Halpern, Victor Kaftal and László Zsidó, Finite weight projections in von Neumann algebras $\ldots \ldots \ldots \ldots \ldots \ldots \ldots \ldots \ldots \ldots$

Telemachos E. Hatziafratis, Explicit $\bar{\partial}$-primitives of Henkin-Leiterer kernels on Stein manifolds

Ka Hin Leung, A construction of an ordered division ring with a rank one

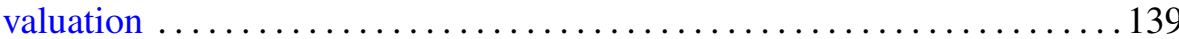

Christopher K. McCord, Nielsen numbers and Lefschetz numbers on solvmanifolds

Katsuro Sakai and Raymond Y. T. Wong, Manifold subgroups of the homeomorphism group of a compact $Q$-manifold

Caroline Perkins Sweezy, $L$-harmonic functions and the exponential square class 